\title{
Review of LoRa WAN and its Application
}

\author{
Mr.Jitendra Rajendra Rana ${ }^{\# 1}$, Dr. S. A. Naveed ${ }^{\# 2}$, \\ MGM's Jawaharlal Nehru Engineering College, \\ N-6, CIDCO, Aurangabad, Maharashtra, India
}

\begin{abstract}
Today entire world is surrounded by wireless signals. IoT applications along with wireless sensors can create useful smart city applications. The conventional wireless options like mobile signals and data transmission \& application specific wireless protocol are playing important role in IoT applications. Wireless technology helps us for Smart home automation, Industry Automation, Automobile automation, specific appliance, and equipment automation. In smart city application which analyzes data, monitor data as well as manage data of urban area, for effective communication proper wireless protocol is basic requirement. At present Bluetooth, ZigBee, $Z$ wave, NFC, RFID, Wi-Fi, LoRaWAN and many more application specific wireless protocol are available in the market. The review latest wireless technology for IoT i.e. LoRaWAN an application specific wireless protocol is proposed in this paper along with comparison.
\end{abstract}

Keywords: Bluetooth, ZigBee, , Wi-Fi, LoRaWAN, LPWAN

\section{INTRODUCTION}

The wireless protocol is a must need thing for implementing any kind of automation. In a wireless sensor network system these wireless protocols are used to transmitting the various parameter signals detected by the sensor as well as revert back the control signals generated by the controller. Smart city is a concept for urban area where using different types of sensors and controllers (IoT) data is collected to manage assets and resources more efficiently. In an urban area wireless sensor network is used to interconnect various physical devices to the internet. The task of the controller in a system is to collect and monitor the information sent by these sensors and generate controlling signals according to the status of the system. In this system data parameters are transmitted and received multiple times through selected wireless protocol.
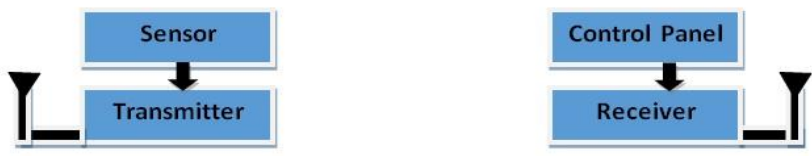

Wireless Transmission

Wireless Transmission

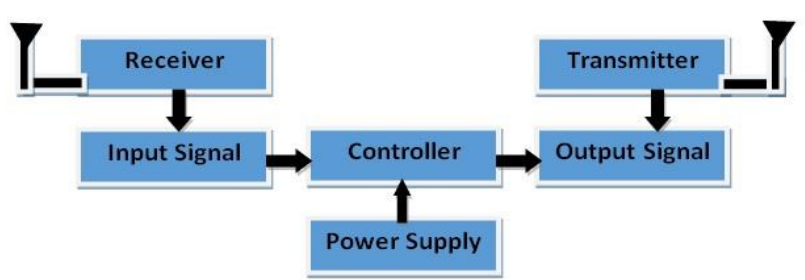

Figure 1. Block diagram of the smart city application system
Sending the data at a long distance using any of the wireless technology plays important role in applications such as smart parking sensors, wireless drones, traffic signal monitoring, automated meter reading, Home and building automation, Wireless alarms, security, and surveillance systems, Industrial monitoring and control. In fact any application that requires data only low power long range wireless transmission will be beneficial for IoT Applications.

The wireless technologies that are used for such application till date are conventional RF transmission techniques using AFSK and ASK, Zigbee and wifi. The data transfer rate for above mentioned technologies is promising \& these technologies do transmit the data over long range but requires large transmission current thereby not being suitable in some battery powered applications. The other option for transmitting the data parameter is GSM module. The drawback of GSM module is the dependency on the network service provider and operator charges. These drawbacks are not suitable for an application of data transfer with low power long range wireless data transmission where devices are operated on battery supply. In recent studies LoRaWAN has shown significance output for preparing such low power network.

\section{FEATURES OF LORAWAN}

Taking example of SEMTECH SX1272 LoRa Transceiver module following are the features of LoRaWAN.

1. Programmable bit rate of $300 \mathrm{kbps}$

2. Operating temperature range -40 to +85 degree.

3. Frequency Range of $860-1020 \mathrm{MHz}$.

4. Spreading factor 6-12

5. Bandwidth $125-500 \mathrm{KHz}$

6. Sensitivity -117 to $-137 \mathrm{dBm}$

7. MLQP 28 pins lead package

8. Supply voltage $1.8 \mathrm{~V}$ to $3.7 \mathrm{~V}$

9. Integrated synthesizer with resolution of $61 \mathrm{~Hz}$

10. LoRa Modem.[6]

\section{PROPOSED METHODOLOGY}

In recent development many research have been proposed based on LoRaWAN and its application for smart city. To identifying the gap in present research and overcome it with suitable solution is proposed in this article. Further development for smart city application will be achieved using observation and comparison. 


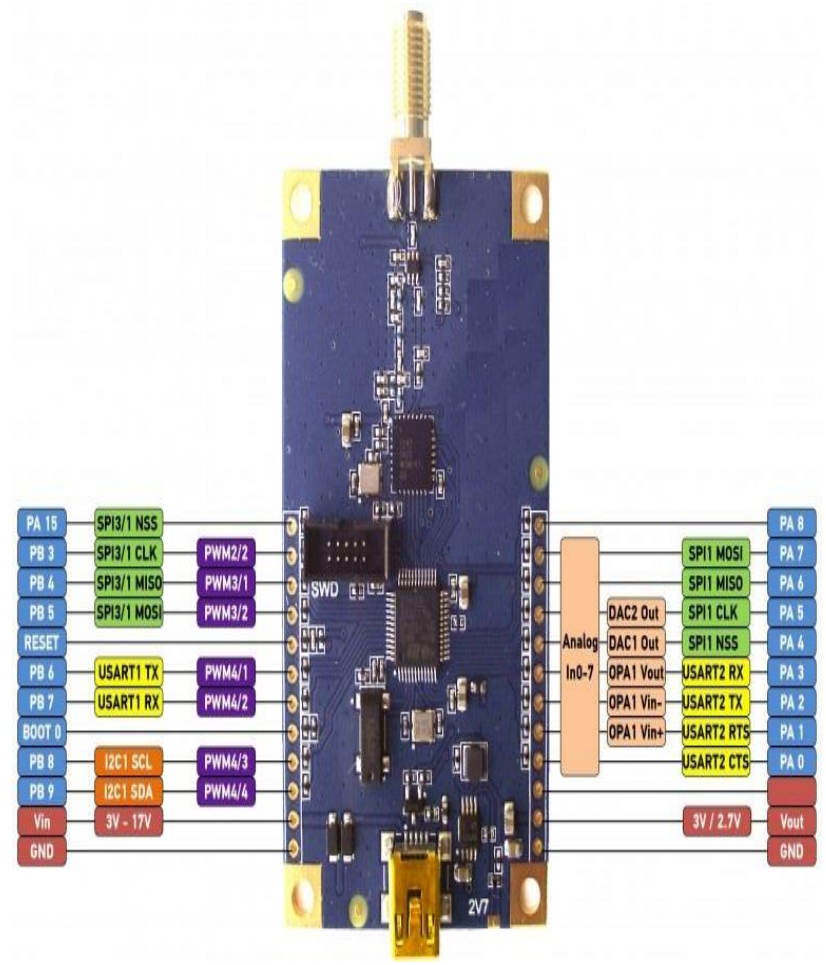

Figure 2. LoRa 1272 Module [6]

- LoRaWAN based weather monitoring system.

The system was proposed 2018 to represent the feasibility of implementation of LoRaWAN for weather monitoring in remote areas of Malaysia. As per the proposed system all weather monitoring data was transmitted up to $23 \mathrm{~km}$ with $7.3 \mathrm{dBm}$ speed. Battery utilization was effectively improved for this system. [1]

- Smart Energy meter using LoRaWAN.

The system was proposed by the author for consumer metering and it discuss about implementation of smart energy meter using LoRaWAN. The proposed system has used LoRaWAN effectively up to $5 \mathrm{~km}$ range for obtaining voltage, current, frequency and other related parameters over internet using IoT. No battery utilization parameter was mentioned. [2]

- Water meter reading system using LoRaWAN In the system proposed by the author for getting water meter reading multilevel relay and concentrator based on LoRaWAN. That proposed system has achieved accurate measurement of water meter data. It has achieved data transmission up to $7^{\text {th }}$ floor of the building. In this system seven times low battery consumption is achieved. [3]

- IoT based Health monitoring System.

In this proposed system medical sensors are integrated with LoRaWAN using internet of things. Blood pressure, sugar level, body temperature data is collected of a human body. This system has achieved data transfer in a radius of $33 \mathrm{sqm}$ more so over ten times low battery consumption is achieved. [4]
- Vehicle monitoring system using LoRaWAN.

The proposed system was used for urban area to identify and monitor air pollution and climate changes. The vehicle is used to collect data from different location in city area. Many environmental parameters like ambient temperature and humidity, and air quality parameters, including $\mathrm{PM} 2.5, \mathrm{NO} 2, \mathrm{CO}$, and $\mathrm{O} 3$ were monitored using this system. That system has provided GPS positions of the vehicle, weather parameters in different areas, vehicle information, and air quality information.

\section{LITERATURE OVERVIEW}

The literature review of all paper that is studied indicates that LoRaWAN is a useful low power protocol for implementing smart city applications on a large scale. Following are the key aspects of LoRaWAN which are,

- Low power data transfer.

- Improvement in sensor battery life.

- Long range data transfer.

- Wide application area.

- Cost effective.

- Secure data transfer.

- Centralized control unit.

- Implementation in all network topologies.

- Overcome disadvantages of other topologies.

There are some factors which also need to be considered while designing a system using LoRaWAN.

- Low Data transfer rate.

- Implementation in complex application is unknown.

- Sensor and LPWAN interface.

- Interference in data signal.

- Overall speed of data transfer for fast response circuitry.

- Bluetooth

\section{COMPARISON}

- Bluetooth is IEEE 802.15.1 wireless protocol.

- Operating frequency is $2.400 \mathrm{GHz}$ to $2.485 \mathrm{GHz}$.

- Low Power consumption protocol.

- Range limitation up to100 meters.

- Data transfer rate up to $24 \mathrm{Mbps}$.

- Useful for low range data transfer.

- Wi-Fi Wi-Fi is IEEE 802.11 wireless protocol.

- Operating frequency $2.4 \mathrm{GHz}$ to $5.0 \mathrm{GHz}$

- High power consumption protocol.

- Limited range up to 150 meters in radius.

- Data transfer rate $3.46 \mathrm{Gbps}$.

- Useful for low range data transfer.

- Useful for wide range data transfer.

- ZigBee

- ZigBee IEEE 802.15.4 wireless protocol.

- Operating frequency $2.4 \mathrm{GHz}$.. 
- Low power consumption protocol.

- Range up to 1500 meters in line of sight.

- Data transfer rate $1 \mathrm{Mbps}$.

- Useful for long range data transfer.

- LoRaWAN

- LoRaWAN is IEEE 802.15 wireless protocol.

- Operating frequency $433 \mathrm{MHz}, 868 \mathrm{MHz}$, $915 \mathrm{MHz}$.

- Low power consumption protocol.

- Range up to $10 \mathrm{Km}$ in rural area.

- Data transfer rate 50kbps.

\section{RESULTS ANALYSIS}

Comparison result indicates following aspects of wireless protocol and for low power application such as data reception from sensor, LoRa is preferable.

Table No.1: Result Analysis

\begin{tabular}{|l|l|l|}
\hline $\begin{array}{l}\text { Sr. } \\
\text { No. }\end{array}$ & $\begin{array}{l}\text { Wireless } \\
\text { Protocol }\end{array}$ & $\begin{array}{l}\text { Observations for smart city low } \\
\text { power long range data transfer } \\
\text { application. }\end{array}$ \\
\hline 1 & Bluetooth & $\begin{array}{l}\text { Range is limited. Not Suitable for } \\
\text { smart city applications. }\end{array}$ \\
\hline 2 & Wi-Fi & $\begin{array}{l}\text { Moderate range but power } \\
\text { consumption is more. }\end{array}$ \\
\hline 3 & ZigBee & $\begin{array}{l}\text { Suitable for long range application } \\
\text { but needs more number of repeaters. }\end{array}$ \\
\hline 4 & LoRaWAN & $\begin{array}{l}\text { Low Power long range data transfer } \\
\text { possible effectively. }\end{array}$ \\
\hline
\end{tabular}

\section{CONCLUSION}

LoRaWAN protocol is having huge scope for implementation of smart city application. More research in this area is necessary to obtain effective solutions for smart city applications. As per the current comparison LoRaWAN is beneficial over WiFI and ZigBee. Other similar technologies like DASH7, Sigfox, weightless will be compared further to identify best LPWAN for smart city application.

\section{REFERENCES}

[1] N.H.Abd Rehman, Y Yamada, M H Husni, N.H.Abdul Aziz, "Analysis of propagation link for remote weather monitoring system through LoRa Gateway", 2nd International Conference on Telematics and Future Generation Networks, 2018.

[2] Nisargh Shah, S Sundar, "Smart Electric Meter using LoRa protocol and IoT applications", IEEE 2nd International conference on Electronics, Communication and Aerospace Technology, 2018.

[3] Yuezhong Li, Lingyuan Zeng , Hualing Wu,

Xiaoqiang Yan, "Research on Water Meter Reading System Based on LoRa Communication", IEEE International Conference on Smart Grid and Smart Cities 2017.

[4] Afef Mdhaffar, Tarak Chaari, Kaouthar Larbi Mohamed Jmaiel and Bernd Freisleben, "IoT-based Health Monitoring via LoRaWAN", IEEE EUROCON, 2017

[5] Chao-Linag Hsieh, Zheng-Wei Ye, Chen-Kang Huang, YeunChung Lee, Chih-Hong Sun, Tzai-Hung Wen, Jehn-Yih Juang, Joe-Air Jiang S. "A Vehicle Monitoring System Based on the LoRa Technique", World Academy of Science, Engineering and Technology International Journal of Transport and Vehicle Engineering Vol:11, No:5, 2017

[6] Semtech wireless and sensing product datasheet for SX1272/1273, Semtech semiconductor Ltd.

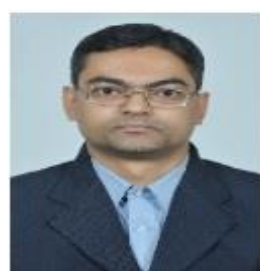

Dr. Syed A. Naveed

$\mathrm{He}$ is Faculty in Jawaharlal Nehru Engineering College, Aurangabad (M.S.), India. He has completed B.E., M.E., Ph.D.(EEE), LL.B. and M.B.A.(HR) in 2000, 2001, 2009, 2015 and 2018 respectively. He has more than 15 years of experience in technical field. He has 40 national/ international technical publications. He is Chartered Engineer with IE and Chartered Industrial Environmentalist with SES. He has been presented \&quot; Rajiv Gandhi Excellence Award \& quot; on 24-08-2013 and conferred with \&quot; The Best Citizens of India Award\& quot; in 2013. He is life member of various 77 national/international professional bodies. His areas of interest are power system, power quality, power electronics and renewable energy.

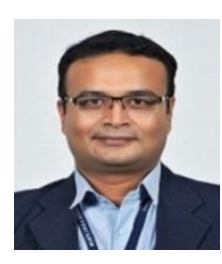

Prof. J. R. Rana

$\mathrm{He}$ is research scholar and faculty in Jawaharlal Nehru Engineering College, Aurangabad (M.S.), India. He has completed B.E., M.E. Electronics in 2004 and 2010 respectively. He has more than 13 years of experience in technical field. He has 16 national/ international technical publications. He is life member of IAENG, IFERP professional bodies. His areas of interest are Wireless sensor network, microcontroller applications, digital electronics, digital signal processing. 